\title{
Anatomy of On-Surface Synthesized Boroxine 2D Polymers
}

Nerea Bilbao, ${ }^{* a}$ Cristina Martín,a,b, Gaolei Zhan,a, ${ }^{,}$Marta Martínez-Abadía, ${ }^{c}$ Ana Sanz-Matias, ${ }^{d}$ Aurelio Mateo-Alonso, ,e Jeremy N. Harvey, ${ }^{d}$ Mark Van der Auweraer, ${ }^{a}$ Kunal S. Mali, ${ }^{*} a$ and Steven De Feyter*a

aDepartment of Chemistry, Division of Molecular Imaging and Photonics, KU Leuven, Celestijnenlaan 200F, B-3001 Leuven, Belgium; bDepartamento de Química Física, Facultad de Farmacia, Universidad de Castilla-La Mancha, 02071 Albacete, Spain; cPOLYMAT, University of the Basque Country UPV/EHU. Avenida de Tolosa 72, E-20018 Donostia-San Sebastián, Spain; dDepartment of Chemistry, Quantum Chemistry and Physical Chemistry, KU Leuven, Celestijnenlaan 200F, B-3001 Leuven, Belgium; eIkerbasque, Basque Foundation for Science, Bilbao, Spain.

Corresponding authors: nerea.bilbao@kuleuven.be, kunal.mali@kuleuven.be, steven.defeyter@kuleuven.be

\begin{abstract}
:
Synthetic 2D polymers (2DPs) obtained from well-defined monomers via bottom up fabrication strategies are promising materials that can extend the realm of inorganic 2D materials. The on-surface synthesis of such 2DPs is particularly popular, however the pathway complexity in the growth of such films formed on solid surfaces is poorly understood. In this contribution, we present a straightforward experimental protocol which allows the synthesis of large area, defect-free 2DPs based on boroxine linkages at room temperature. We focus on unravelling the multiple pathways available to the polymerizing system for the spatial extension of the covalent bonds. Besides the anticipated 2DP, the system can evolve into self-assembled monolayers of partially fused monodisperse reaction products that are difficult to isolate by conventional synthetic methods, or remain in the monomeric state. The access to each pathway can be controlled via monomer concentration and the choice of the solvent. Most importantly, the unpolymerized systems do not evolve into the corresponding 2DP upon annealing, indicating the presence of strong kinetic traps. Using high resolution scanning tunneling microscopy (STM), we show reversibility in the polymerization process where the attachment and the detachment of monomers to 2DP crystallites could be monitored as a function of time. Finally, we show that the way the 2DP grows depends on the choice of the solvent. Using UV-Vis absorption and emission spectroscopy, we reveal that the dominant pathway for 2DP growth is via in-plane self-condensation of the monomers whereas in the case of an aprotic solvent the favored growth mode is via $\pi$ stacking of the monomers.
\end{abstract}

KEYWORDS: two-dimensional polymers, scanning tunneling microscopy, on-surface synthesis, infrared spectroscopy, UV-visible spectroscopy 
A two-dimensional polymer (2DP) is a single molecule thin, structurally precise, sheet-like macromolecule that consists of laterally linked repeat units connected via covalent bonds. These macromolecules are a subset of layered (2D) materials, with graphene being the quintessential 2D material. A 2DP thus represents a "designer" variant of graphene which can be synthesized in a truly bottom-up approach using established principles of synthetic organic chemistry. These customized 2D materials have potential applications as membranes for gas separation and water desalination, and also as active materials in transistors and sensors. ${ }^{1,2}$

Typically, a 2DP is synthesized by covalently linking organic building blocks using dynamic covalent chemistry (DCC) which allows harvesting of one desired product out of a combinatorial library under equilibrium conditions. ${ }^{3}$ The two most widely used reactions are the Schiff base formation ${ }^{4}$ and (self)condensation of boronic acids.5, 6 2DPs based on boronate ester linkages are also popular.7, 8 Since water is released during these condensation reactions, it is possible to maintain equilibrium conditions and thus reversibility, by regulating the amount of water present in the system during the polycondensation process. Such reversibility is extremely important for the formation of defect-free, extended domains and remains the hallmark of 2DP synthesis. ${ }^{9}$ Alternatively, irreversible reactions such as Ullmann-type coupling 10 and cycloaddition reactions ${ }^{11}$ which involve carbon-carbon bond formation, have also been employed for 2DP synthesis.

An important sub-class of 2DPs is the so-called 2D covalent organic frameworks (2D COFs) which consist of stacked 2DP sheets. ${ }^{12}$ 2D COFs are typically obtained as microcrystalline powders using solvothermal conditions. The main drawback of the solvothermal synthesis is that the polymer sheets remain stacked and their delamination into single layers is an extremely challenging task. ${ }^{13,14}$ The $2 \mathrm{D}$ COF powders suffer from poor processability, further complicating their molecular level characterization. It is generally accepted that the nanoscale characterization of such low-dimensional materials is a crucial step in the optimization of their functional properties.

The limitations of the solvothermal methods have prompted the exploration of alternative synthetic paradigms for the fabrication and characterization of 2DPs. In this context, the on-surface synthesis of 2DPs, where surfaces are employed as inherently 2D reaction platforms, has gained momentum in the recent past. ${ }^{15}$ The advantage of this approach lies in the fact that the dynamics as well as the reaction between the monomers are already confined in 2D, thus promoting the in-plane growth of the material. Both solid as well as fluid interfaces have been employed for the synthesis of 2DPs which often provide access to single or few layered films. Such films, provided they are formed on/transferred to a (conductive) substrate, are then amenable to molecular level characterization using scanning tunneling microscopy (STM) and non-contact atomic force microscopy (AFM).16-22 Recently, tip-enhanced Raman spectroscopy (TERS) has also been employed for obtaining nanometer scale chemical information on the nature of defects present in 2DP sheets formed at the air-water interface. ${ }^{23}$ 
Despite the encouraging results reported so far, the published literature is mainly limited to fabrication of a given target structure and its molecular level characterization using STM. The on-surface formation of 2DPs remains poorly understood largely because the effective reaction conditions are often arrived at via empirical screening of the building blocks and/or the reaction conditions. Given the multiple pathways available for the spatial extension of the covalent bonds, the polycondensation process often involves generation of multiple covalent structures with varying free energies. This pathway complexity remains virtually unaddressed. An in-depth understanding of these aspects is key to the optimization of reaction conditions which in turn is critical for controlling the crystallinity, morphology and thus the overall quality of the 2DP films. A survey of the recent literature reveals that the design aspect of this research has significantly outpaced the complementary fundamental studies which are essential for optimizing the 2D polymerization process. Investigation of the mechanistic aspects of COF growth, especially gaining insight into the 2D polymerization and crystallization of bulk 2D COFs, is an emerging area of research. Some recent reports have already provided important insight into the polymerization and crystallization process of bulk 2D COFs both experimentally ${ }^{24-27}$ and theoretically ${ }^{28,}$ ${ }^{29}$ and also for on-surface synthesized 2DPs. ${ }^{9}$

In this work, we describe the on-surface synthesis of boroxine-based, defect-free 2DPs at the solution-graphite interface and at room temperature. We focus on unravelling the mechanistic aspects and demonstrate the presence of multiple pathways in the on-surface $2 \mathrm{D}$ polymerization process leading to different structural outcomes. The access to each pathway can be precisely controlled by the choice of experimental parameters such as the monomer concentration and the choice of the solvent. Besides the expected 2DP, the formation of thin films, which consist of self-assembled networks made up of either monomers or partially fused monodisperse reaction products was observed. Furthermore, the experimental data reveals the presence of strong kinetic traps that prevent the evolution of some of these structures into the final 2DP. The surface-confined films were characterized in detail using STM, AFM and attenuated total reflection Fourier transform infrared (ATR FT-IR) spectroscopy. We also took initial steps to understand the difference in the nucleation of the 2DP depending on the nature of the solvent. A combination of STM measurements and UV-Vis absorption and emission spectroscopy reveal initial nucleation of 2DP crystallites in certain solvents and different modes of growth in solution depending on the choice of the solvent. This study brings mechanistic insight into the process of on-surface 2DP formation, in particular at the solution-solid interface, providing a foundation for the construction of large-area defect-free 2DPs.

Pyrene-2,7-diboronic acid (PDBA) was selected as the building block for this investigation as pyrene-based monomers are routinely used ${ }^{30-32}$ for the synthesis of bulk 2D COFs due to their large $\pi$ surface, which bestows attractive photoconductive properties to the resulting material. ${ }^{33-35}$ Furthermore, the on-surface synthesis of the boroxine 2DP from PDBA has been reported in the recent past. The 

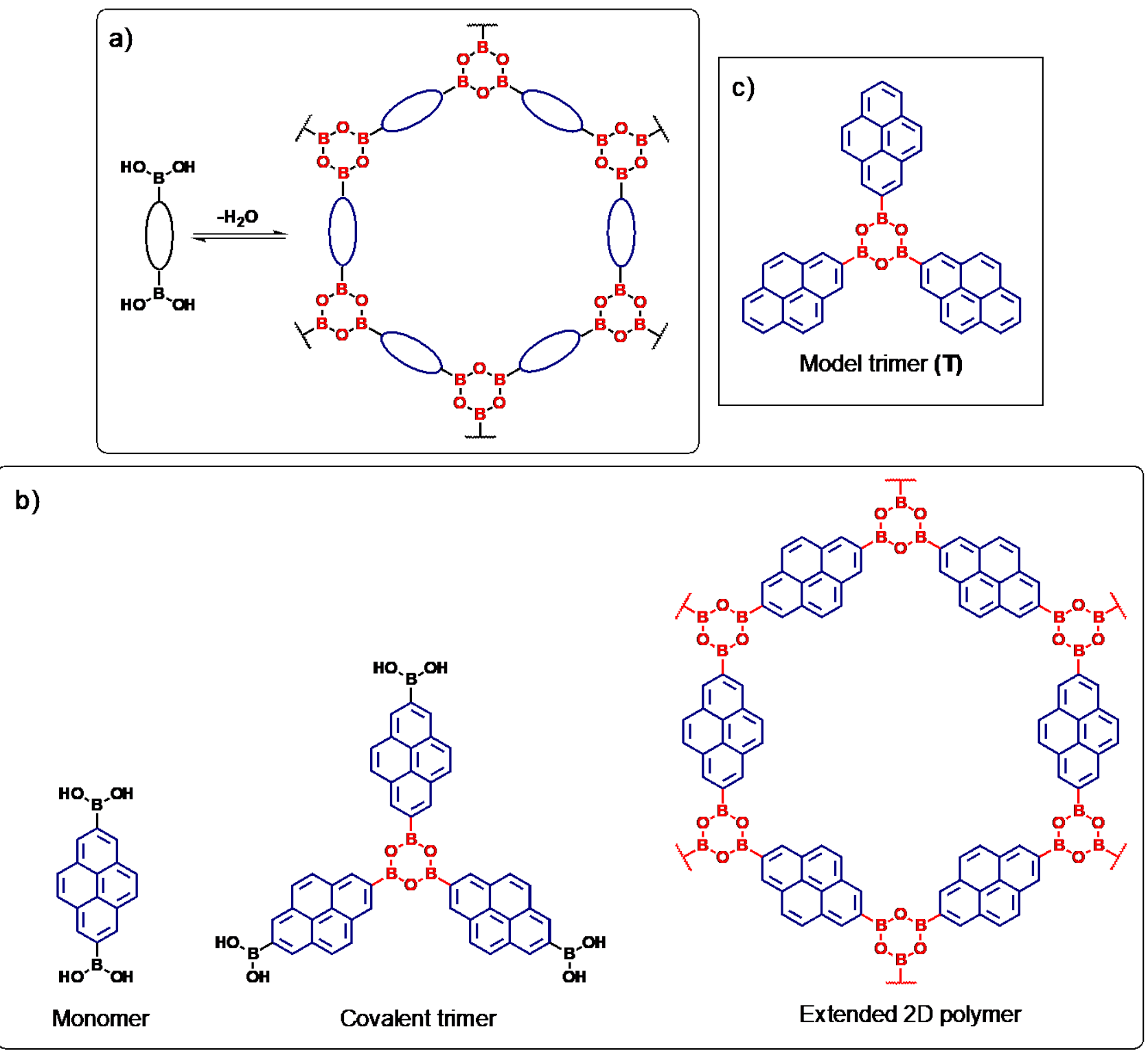

Scheme 1. (a) General reaction scheme showing the self-condensation of aryl diboronic acids to yield 2DP. (b). Molecular structure of the PDBA monomer, covalent trimer and the PDBA 2DP. (c) Molecular structure of the model covalent trimer synthesized independently.

reported protocol consists of heating a suspension of PDBA in heptanoic acid on the surface of highly oriented pyrolytic graphite (HOPG) for $1 \mathrm{~h}$ at $120{ }^{\circ} \mathrm{C}$ in a stainless-steel reactor. It yielded the corresponding 2DP with domains that extend a few tens of square nanometers. ${ }^{19}$ Note that the previous reports describing the on-surface self-condensation of boronic acids employed relatively active substrates such as $\mathrm{Ag}, \mathrm{Cu}$ or $\mathrm{Au}$ and/or moderately elevated $\left(80-100{ }^{\circ} \mathrm{C}\right)$ temperatures..$^{36-38}$ The solvothermal synthesis of boroxine based 2D COFs typically consists of heating the monomer suspension between temperatures of $80-150{ }^{\circ} \mathrm{C}$ in a closed vessel for several hours to several days. 33,39 


\section{RESULTS AND DISCUSSION}

In the present study, solid PDBA was dissolved in dimethyl sulfoxide (DMSO) and this stock solution was subsequently diluted using octanoic acid to prepare a concentration series. This preparation method allows better solubility of the monomers, at least within the concentration range studied here. The importance of maintaining homogeneous conditions, where the monomers are sufficiently soluble in the solvent, has been highlighted for the on-surface ${ }^{40}$ as well as the bulk synthesis of 2D COFs. ${ }^{25}$ Furthermore, good solubility of the monomers also allows monitoring of the dynamic processes that transpire during the on-surface 2D polymerization process using STM.

Figure 1 shows the STM data obtained on thin films after deposition of a series of solutions with increasing monomer concentration. Contrary to the expectation, a network composed of rows of threelobed features was obtained at relatively lower concentrations (Figure 1a). This network is drastically different from the anticipated hexagonal network of the boroxine based 2DP and consists of alternating rows of three lobed features running in opposite direction. We attribute these features to covalent trimers obtained by self-condensation of three PDBA molecules (vide infra) which are further selfassembled via hydrogen bonding interactions between unreacted boronic acid units. A molecular model that reproduces the unit cell obtained from the STM data is presented in Figure 1b. The model reveals open spaces between self-assembled trimers which are possibly occupied by solvent molecules that are too mobile on the time scale of STM imaging. We note that the dimerization of aryl boronic acids via cyclic hydrogen bonding has been well-studied. ${ }^{41}$ Figure S1 in the supporting information shows that a hypothetical, purely hydrogen-bonded trimer would lead to a larger unit cell than that measured here. Apart from the ordered self-assembled network displayed in Figure 1b, the covalent trimers also form other types of assemblies stabilized by hydrogen bonds, however, the surface coverage of such structures is low (Figure S2 in the supporting information). It is worth highlighting that such a reaction product would be difficult to synthesize in solution and using conventional synthetic methods. The ability of surfaces to act as reaction platforms for the synthesis of exotic products has been demonstrated recently. ${ }^{42,43} \mathrm{~A}$ model trimer, hereafter referred to as $\mathbf{T}$ and lacking terminal boronic acid groups (Scheme 1c), was synthesized for the sake of comparison. For this compound however, no self-assembled networks could be detected using STM under different experimental conditions. The lack of stable selfassembled network formation for $\mathbf{T}$ highlights the stabilization offered by the hydrogen bonding interactions between the terminal boronic acid units.

An increase in the concentration of the PDBA monomers in solution yielded a hexagonal network with a periodicity that matches with the anticipated 2DP based on boroxine rings. The defect-free domains often extend over more than $120 \times 120 \mathrm{~nm}^{2}$ (Figure S3 in the supporting information). Figure 1c shows a sub-molecular resolution STM image of the network in which each pyrene unit appears as a six-lobed feature whereas the boroxine rings do not exhibit any STM contrast. Although the 2DP is 

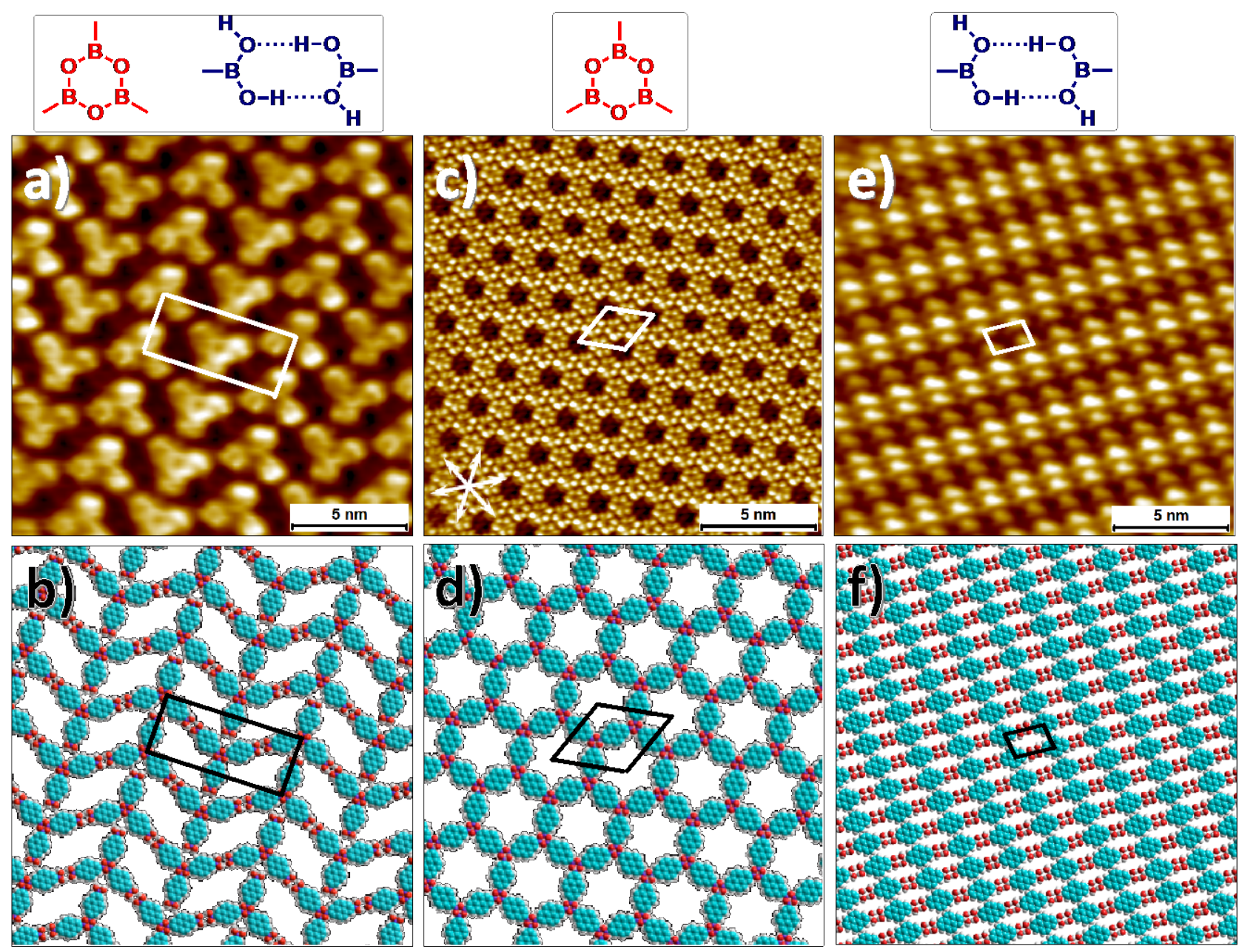

Increasing concentration of monomers

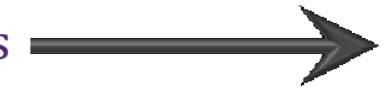

Figure 1. Pathway complexity in the on-surface synthesis of a 2DP based on PDBA as a function of monomer concentration at the octanoic acid-DMSO/HOPG interface. The chemical schemes show the dominant binding mode involved in the formation of each network shown in the STM images presented below. (a) STM image showing a self-assembled monolayer formed by covalent trimers obtained after partial self-condensation of PDBA. Monomer concentration $=1.5 \times 10^{-5} \mathrm{M}(0.45 \% \mathrm{v} / \mathrm{v}$ DMSO). (b) Molecular model for the trimer self-assembled network. (c). STM image of the PDBA 2DP formed at the octanoic acid-DMSO/HOPG interface. Monomer concentration $=1.0 \times 10^{-4} \mathrm{M} .(2.6 \% \mathrm{v} / \mathrm{v}$ DMSO) (d) Molecular model for the PDBA 2DP. (e) Self-assembled network formed by PDBA monomers. Concentration $=3.8 \times 10^{-4} \mathrm{M} .(10 \% \mathrm{v} / \mathrm{v}$ DMSO $)(\mathrm{f})$ Molecular model for the monomer self-assembled network. For large scale STM images see Figure S3, S6 and S7 in the SI.

expected to offer periodic shallow cavities with a diameter of $\sim 1.7 \mathrm{~nm}$, the cavity diameter measured from the STM data is only $\sim 1.3 \mathrm{~nm}$ (Figure S4 in the supporting information). The reason behind this discrepancy is not entirely understood. However, it is important to note that the STM image contrast does not necessarily map the exact van der Waals volume of the structure. The unit cell vector for the 2DP 2.0 
$\pm 0.1 \mathrm{~nm}$, Table 1) however resembles closely with the in-plane lattice constant reported previously for the corresponding bulk framework $(2.22 \mathrm{~nm})$ and our independent calculations (Figure S5 in the supporting information). ${ }^{33}$ A previous report ${ }^{19}$ indicated that the thin films of PDBA formed on the surface of HOPG are in fact bilayers of the 2DP with eclipsed stacking similar to that observed for the corresponding bulk 2D COF.33 The polymer films formed at the octanoic acid-DMSO/HOPG interface in the present case however, appear to be made up of a single layer and there is no evidence of bilayer formation. The seamless formation of the 2DP at RT in the present case could partly be linked to the solvent mixture employed here. DMSO is a stronger proton acceptor than heptanoic acid and small amounts of this solvent could also enhance the dynamic character of the formation of the covalent structures.

At even higher concentrations, a densely packed network is obtained where the PDBA monomers are self-assembled via hydrogen bonding between the boronic acid units. These hydrogen bonded arrays remain stable over time and do not undergo transition into any of the other structures described above. The formation of a well-ordered network of hydrogen bonded PDBA monomers at room temperature is in contrast to a previous study, ${ }^{19}$ where the networks remained disordered even after annealing at $70{ }^{\circ} \mathrm{C}$ for 1 hour in heptanoic acid. This difference could be explained by considering the better solubility of PDBA in DMSO/octanoic acid mixture compared to heptanoic acid alone. The better solubility translates into better dynamics at the solution-solid interface thus allowing formation of well-ordered supramolecular networks.

While the formation of the hydrogen-bonded self-assembled network at higher monomer concentrations appears rather counterintuitive, it can be rationalized by considering the higher extent of kinetic trapping expected under these conditions. We propose that higher monomer concentration leads to formation of a densely packed, hydrogen-bonded supramolecular network faster than it takes for the condensation reaction to proceed to yield large enough areas of the 2DP. It is readily understood that the barrier for reversible bond formation is expected to be significantly lower for the formation of a hydrogen-bonded self-assembled network as compared to the covalently bonded 2DP network favoring the formation hydrogen-bonded self-assembled networks over the covalent 2DP.Once the surface is fully covered with the dense network, there plausibly occurs little or no in-plane monomer dynamics that is critical for the formation of large area 2DP.

Table 1. Unit cell parameters of the networks formed by PDBA at the octanoic acid/HOPG interface.

\begin{tabular}{lccc}
\hline \multirow{2}{*}{ System } & \multicolumn{3}{c}{ Unit cell parameters } \\
\cline { 2 - 4 } & $\mathrm{a}(\mathrm{nm})$ & $\mathrm{b}(\mathrm{nm})$ & $\gamma\left({ }^{\circ}\right)$ \\
\hline PDBA trimer assembly & $2.4 \pm 0.2$ & $4.6 \pm 0.1$ & $90 \pm 1$ \\
\hline PDBA-2DP & $2.0 \pm 0.1$ & $2.0 \pm 0.1$ & $60 \pm 2$ \\
\hline PDBA monomer assembly & $1.2 \pm 0.1$ & $0.8 \pm 0.1$ & $79 \pm 1$ \\
\hline
\end{tabular}


The thin films obtained after the evaporation of the solvent were further characterized using ATRIR to obtain a chemical signature of the 2DP formation. Due to the strong absorption from HOPG that interferes with the measurements, the ATR-IR characterization of the thin films was carried out using mica as the substrate (see Figure S8 in the supporting information). The corresponding bulk PDBA 2D COF was synthesized (see supporting information) using a reported solvothermal method for the sake of comparison. ${ }^{33}$
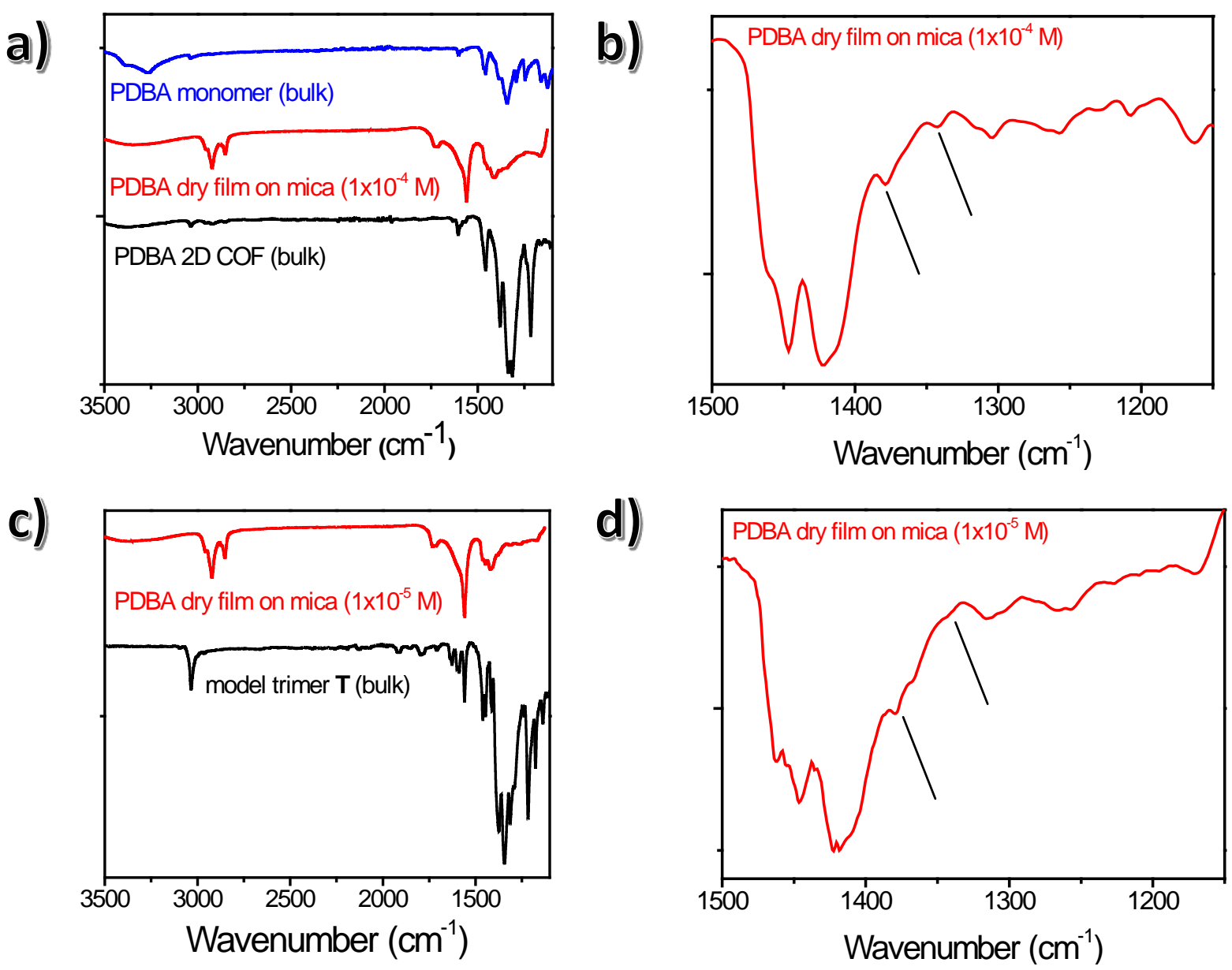

Figure 2. IR analysis of the thin films. (a) Comparison between the IR spectra obtained on PDBA monomer (bulk), PDBA dry film obtained after evaporation of octanoic acid-DMSO $\left(1 \times 10^{-4} \mathrm{M}\right)$ solution on mica and FT-IR spectra of the corresponding bulk 2D COF. Note that the deposition of $1 \times 10^{-4} \mathrm{M}$ PDBA solution in octanoic acid-DMSO on HOPG yields the 2DP. (b) Spectral region from the ATR-IR spectra between 1500 to $1150 \mathrm{~cm}^{-1}$ obtained on the dry films. (c) Comparison between the FTIR spectrum of the model trimer $\mathbf{T}$ and the PDBA dry film obtained after evaporation of octanoic acid-DMSO $\left(1 \times 10^{-5} \mathrm{M}\right)$ solution on mica. Note that at this concentration, a self-assembled monolayer of the covalent trimer is obtained on the surface of HOPG. (d) Spectral region from the ATR-IR spectra between 1500 to $1150 \mathrm{~cm}^{-}$ 1 obtained on the dry films. 
Figure 2a shows the comparison between the IR spectrum obtained on a thin dry film formed after evaporation of a $1.0 \times 10^{-4} \mathrm{M}$ solution of PDBA in octanoic acid-DMSO mixture, the IR spectrum of the corresponding bulk 2D COF and that of the PDBA monomer. Due to the significantly lower amount of material deposited in the thin films, the intensity of the IR signals is reduced for the films compared to the bulk spectra. The IR spectra of the dry film and the bulk PDBA COF show strong attenuation of the hydroxyl stretching modes observed for the PDBA monomer at around $3267 \mathrm{~cm}^{-1}$. The hydroxyl signal did not disappear for the thin film of the 2DP as well as for the bulk 2D COF. In fact, the O-H stretch signals are not expected to disappear completely from the IR spectra of the corresponding polymeric material as their domain borders are inevitably composed of free boronic acid units. Furthermore, we expect that these dry films also contain free residual monomers which could also contribute to the $\mathrm{O}-\mathrm{H}$ stretching signals. Figure $2 \mathrm{~b}$ shows the ATR-IR spectrum of the thin film deposited on mica highlighting the region between $1500 \mathrm{~cm}^{-1}$ and $1150 \mathrm{~cm}^{-1}$. The bands at $1378.3 \mathrm{~cm}^{-1}$ and $1342.8 \mathrm{~cm}^{-1}$ correspond to the B-O stretching vibrations characteristic for the boroxine rings as reported earlier for COF-1.44

A similar comparison was carried out between the ATR-IR spectra of the thin films formed from $1.0 \times 10^{-5} \mathrm{M}$ solution of PDBA in octanoic acid-DMSO mixture and the FT-IR spectrum of the model trimer T (see Scheme 1c), where the $\mathrm{O}-\mathrm{H}$ stretching mode is absent. Both the diagnostic peaks reported for boroxines are present in the IR spectra of the thin films although their intensity is relatively low. The comparison between the IR data for the thin films and the bulk/model systems indicates the formation of boroxine rings on the surface and supports the formation of the PDBA 2DP and the self-assembled monolayer of the covalent trimer films as concluded from the STM data.

The observation of the two self-assembled networks based on covalent trimers and monomers (Figures 1a and 1e, respectively), is rather peculiar and merits special attention. Self-assembled networks of aryl diboronic acids have been described earlier as intermediate structures en route to the final 2DPs. Terphenyl boronic acid and quaterphenyl diboronic acid both form hydrogen-bonded self-assembled networks at the heptanoic acid/HOPG interface. These networks could be converted to the corresponding 2DPs upon annealing the system to $100{ }^{\circ} \mathrm{C} .{ }^{19}$ However, in the present case, annealing of self-assembled networks (both monomer and trimer) to higher temperatures did not lead to the formation of the 2DP. For example, annealing the HOPG surface covered by the covalent trimer monolayer to $50{ }^{\circ} \mathrm{C}$ for 1 hour, did not yield any change in the network structure (Figure S9 in the supporting information). After annealing the same sample further at $100{ }^{\circ} \mathrm{C}$ for an additional hour no organized structure could be observed on the surface. These experiments indicate that once formed, these networks are sufficiently stable on the surface under given experimental conditions and the thermal activation provided to the system is insufficient to overcome the cohesive forces holding the self-assembled systems together. The high stability of these self-assembled systems could be understood by considering the high enthalpic gain arising from the adsorption energy $(\sim 25 \mathrm{kcal} / \mathrm{mol})^{45}$ of the pyrene units on the HOPG surface, the hydrogen-bonding interactions between the boronic acid units (dimerization energies range from -7.0 to 
$-10.8 \mathrm{kcal} / \mathrm{mol}$, see supporting information, also in agreement with the literature $)^{46}$ and the dense molecular packing within these networks.

Despite the high stability of the networks, which appears to prohibit the transition of the densely packed networks into the 2DP, the PDBA molecules are sufficiently mobile at room temperature and at appropriate concentrations to allow monitoring of the dynamic processes involved in the polymerization at the solution-solid interface. Figure 3 shows a sequence of STM images obtained in the same area over $\sim 15$ minutes. While the time sequence shows significant changes as a whole within the scanned area, the changes occurring in the vicinity of the domain edge of the polymer are most interesting. The image
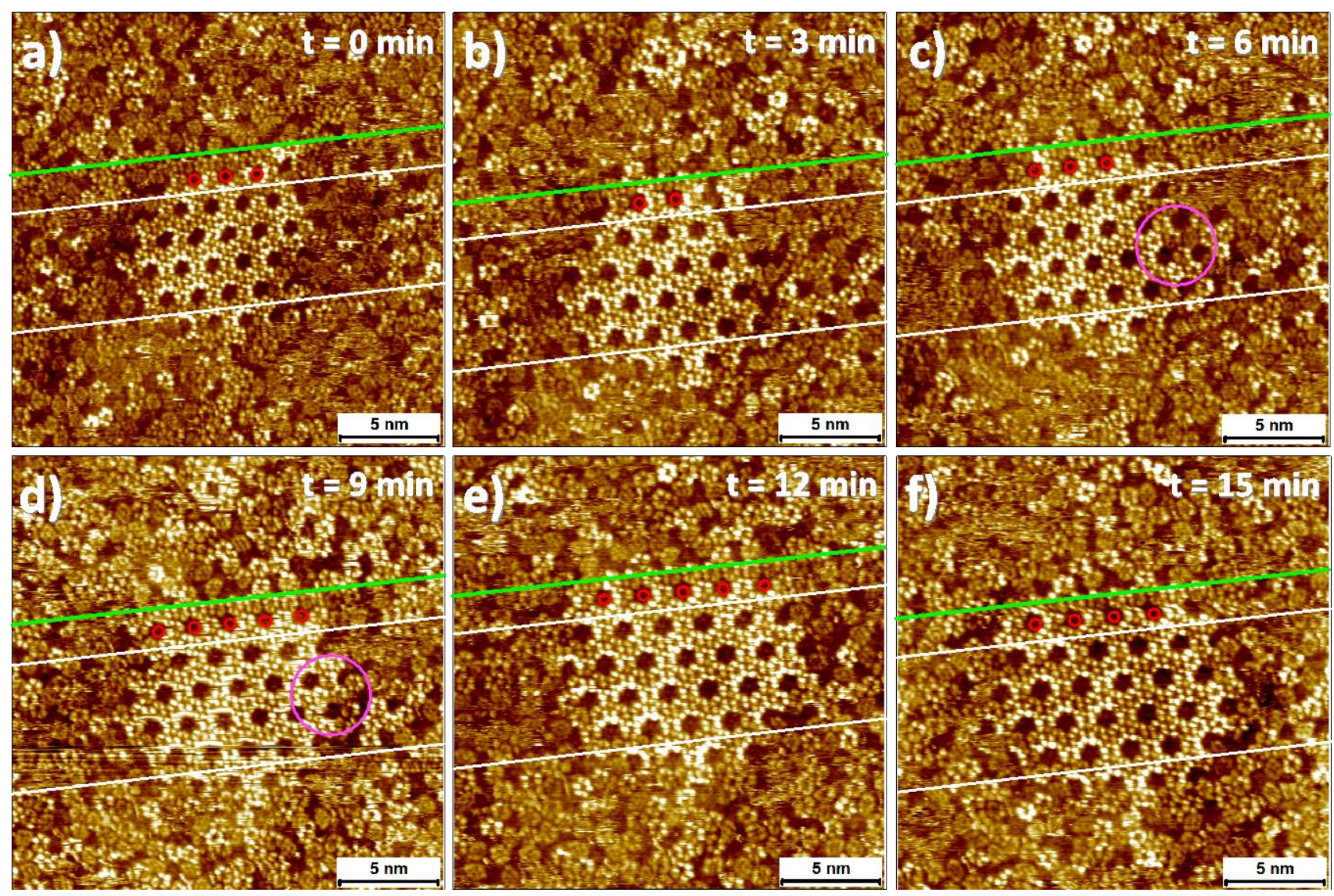

Figure 3. Dynamics of 2D polymerization at the solution-solid interface. [PDBA] $=5 \times 10^{-5} \mathrm{M}$. (a-f) A sequence of STM images recorded in the same area showing the changes in the vicinity of a small 2DP island. The white lines can be used as a general reference to understand the changes in the shape of the island. The attachment and detachment of monomers to the leading edge of the polymer island can be monitored by surveying the area between the white and the green lines, and the red circles which highlight the completely formed cavities. The magenta circles in (c) and (d) highlight the change in the appearance of the PDBA molecules before and after the attachment to the polymer island. 
sequence shows an island of the PDBA 2DP surrounded by randomly oriented PDBA monomers. A noticeable feature is the time-dependent attachment and detachment of monomers to the polymer island which can be followed by comparing the leading edge of the island between the white and green lines (upper part of each STM image). The hollow red circles highlight completely formed cavities. It can be readily seen from the image sequence that there is significant re-arrangement within the leading edge of the polymer. While the first three STM scans (Figure 3a-c) show small change within this area, the polymer seems to grow in subsequent scans (Figure 3d-f).

Another interesting aspect noticeable from the STM images presented in Figure 3 is the different appearance of the PDBA molecules. Within the disordered pool of molecules surrounding the polymer island, the PDBA molecules appear either as six lobed features or as a rhombic shape with four small dark lobes. The pyrene units that are a part of the polymer island however always appear as six lobed features. STM simulations carried out on single molecules of PDBA on the surface of graphite clearly reveal that the difference in the STM contrast of the molecules is related to their adsorption site on the HOPG lattice. The molecules appear as six-lobed features when the stack between the pyrene core and the graphite lattice resembles Bernal stacking whereas a slight parallel displacement of the pyrene core with respect to the graphite lattice results in the STM contrast changing to rhombic shaped features (Figure S10 in the supporting information). Note that the pyrene units that become a part of the polymer island always appear as six lobed features and thus it can be concluded that the polymer has a very specific registry with the underlying substrate lattice. In fact, the appearance of the molecule often changes upon attachment to the island. In the area highlighted by magenta circles, Figure 3c, d, a rhombic shaped feature adjacent to the polymer can be seen to change into a six-lobed feature upon attachment to the island.

The outcome of the polymerization process was found to differ drastically when a non-protic, relatively less polar solvent such as 1,2,4-trichlorobenzene (TCB) was used. Upon dropcasting a freshly prepared, relatively concentrated $\left(1.0 \times 10^{-4} \mathrm{M}\right)$ TCB-DMSO solution of PDBA onto HOPG, a densely packed self-assembled network similar to that observed in octanoic acid-DMSO at the highest concentration of PDBA (see Figure 1e), was observed (Figure 4a). Note that, at this concentration, the 2DP is the dominant phase when octanoic acid-DMSO mixture is used as the solvent. No covalent trimers or other structures were ever detected from diluted solutions in TCB. The unit cell for the self-assembled PDBA network obtained from TCB-DMSO solutions is comparable to that measured for the networks obtained from octanoic acid-DMSO solutions. When the monomer networks were annealed further at 50 ${ }^{\circ} \mathrm{C}$ for $1 \mathrm{~h}$, no self-assembled networks were observed. While no 2DP formation was observed from freshly prepared solutions of PDBA in TCB-DMSO mixture, deposition of a four-week old 1.0 × 10-4 M TCB-DMSO solution onto HOPG at room temperature revealed isolated2DP islands that range in size from about 250 $\mathrm{nm}^{2}$ to $1300 \mathrm{~nm}^{2}$ (Figure 4b). These observations point towards the possibility of the nucleation of small 2DP flakes in the solution phase upon aging of the TCB-DMSO solutions. The small domains of the as- 
deposited 2DP are defect-free. In contrast to the monomer self-assembled networks, which did not yield the 2DP upon annealing, the 2DP crystallites deposited onto the HOPG surface evolved into extended domains of 2DP upon annealing the surface at $50{ }^{\circ} \mathrm{C}$ for 5 minutes (Figure S13 in the supporting information). This indicates that the small 2DP crystallites present on the HOPG surface act as seeds for the growth of the large area 2DP upon annealing. Furthermore, the size of such isolated crystallites was found to increase with increasing aging time of the TCB-DMSO solutions (Figure S14 in the supporting information). We note that such aging behavior was never observed for PDBA solutions in octanoic acidDMSO, indicating that the reversibility of the $\mathrm{B}-\mathrm{O}$ bond formation is enhanced in the protic solvent namely, octanoic acid. A comparison between the results obtained in the two solvents indicates that while boronic acid condensation proceeds in protic as well as aprotic solvents, the pathway followed in each solvent is not the same.
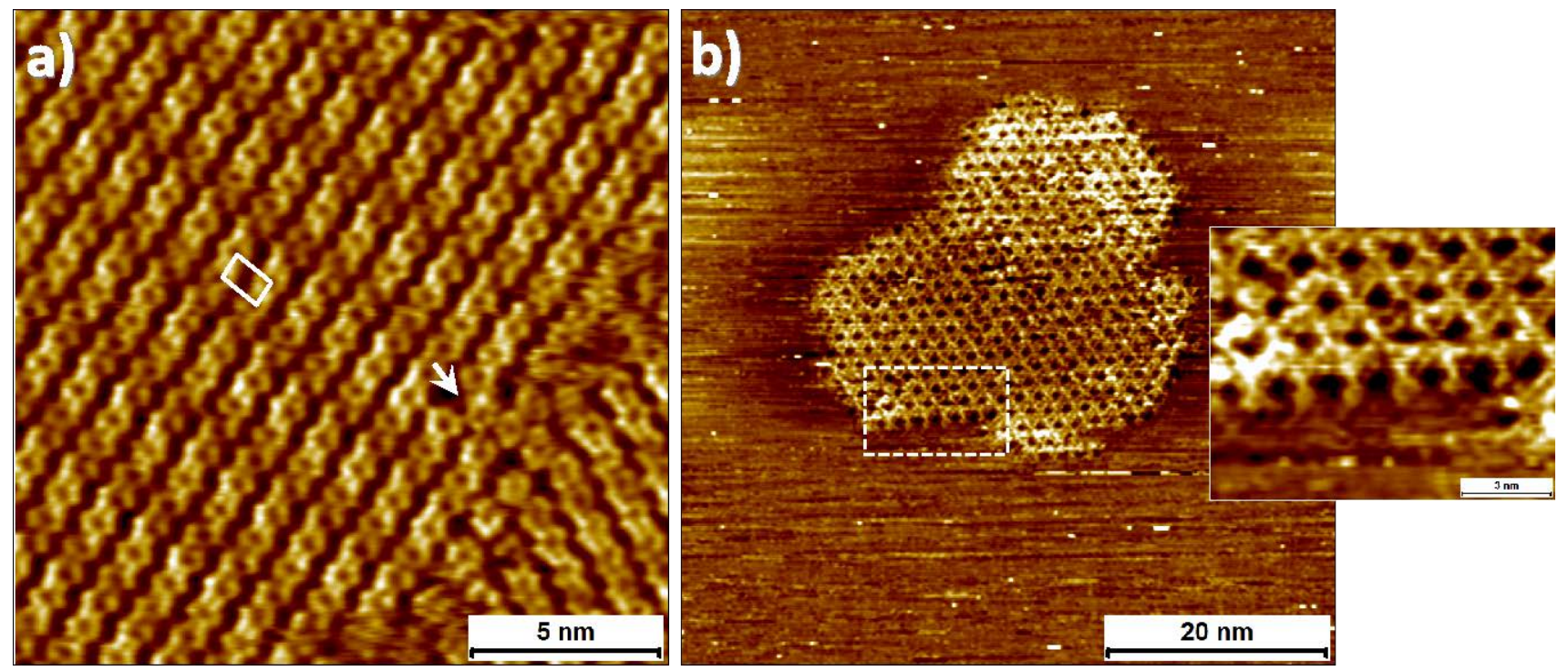

Figure 4. PDBA polymerization from TCB-DMSO solutions. (a) STM image showing the self-assembled monolayer of the PDBA monomers at the TCB-DMSO/HOPG interface. [PDBA] $=1.0 \times 10^{-4} \mathrm{M}(2.6 \% \mathrm{v} / \mathrm{v}$ DMSO). The white arrow shows a defect in the monolayer. (b) STM image showing isolated flakes of the 2DP obtained on the surface of HOPG after deposition of aged (4 weeks) TCB-DMSO solution. [PDBA] = $1.0 \times 10^{-4} \mathrm{M}$. The inset shows a magnified region from the edge of the polymer possibly showing the unreacted ends of the PDBA monomers bearing free boronic acid groups. For additional large-scale images see Figure S11 and S12 in the supporting information.

In order to further understand the growth behavior of PDBA 2DP in solution, UV-Vis absorption and emission spectroscopy was employed. Pyrene is widely known to exhibit excimer emission wherein under certain experimental conditions, it undergoes aggregation in the excited state. This is usually accompanied by a decrease in the fluorescence quantum yield and is associated with an emission at longer wavelengths. It is well-documented that such emission is often devoid of any fine structure. ${ }^{47}$ 
Figures 5a shows the normalized UV-Vis steady-state absorption spectra of PDBA in octanoic acid-DMSO and TCB-DMSO solvent mixtures. For both solutions, the absorption spectra show wellresolved vibrational progressions with a strong absorption band which corresponds to the $S_{0} \rightarrow S_{2}$ transition (338 nm for octanoic acid and $342 \mathrm{~nm}$ for TCB). These absorption spectra are in agreement with previous reports where it was observed that for the 2,7-functionalized derivatives, the $S_{0} \rightarrow S_{2}$ transition is shifted negligibly with respect to the same transition in the case of pyrene. ${ }^{48}$ The small red shift in TCB compared to octanoic acid is due the increased polarizability of TCB. Furthermore, the $\mathrm{S}_{0} \rightarrow \mathrm{S}_{1}$ transition ( $~ 395 \mathrm{~nm}$ for octanoic acid and TCB samples) in the case of 2,7-substituted pyrene derivatives is more favored than in pyrene as shown by their much higher extinction coefficients (Table S1 in the supporting information).
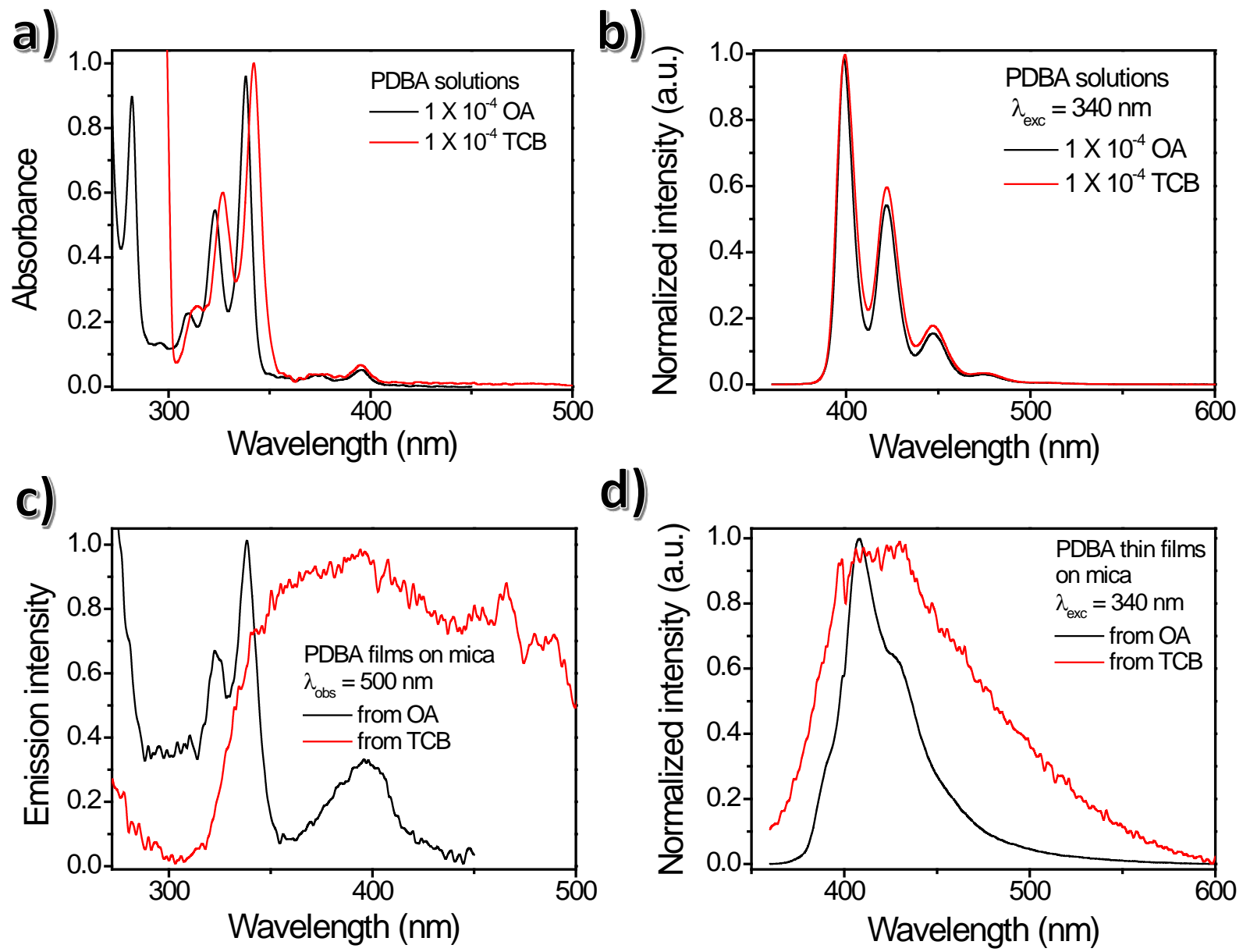

d)

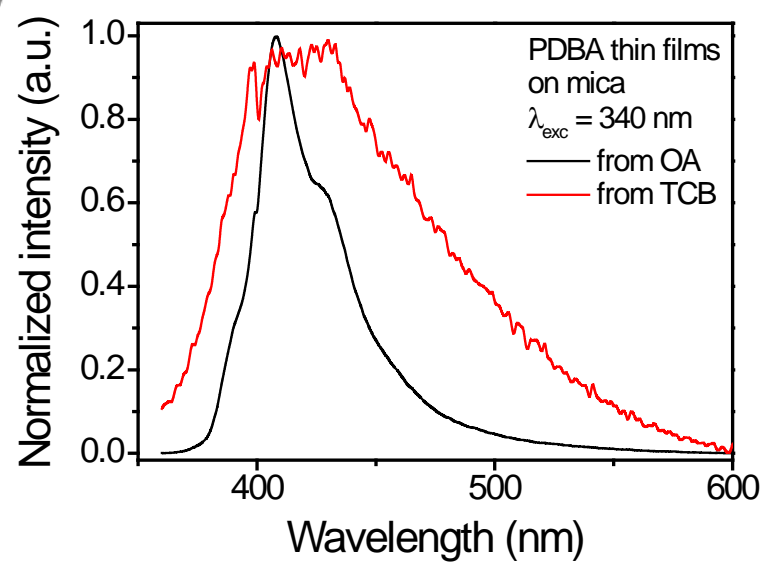

Figure 5. Characterization of the monomer solutions and thin films deposited on mica using UV-vis absorbance and emission measurements. (a) Absorption spectra of PDBA solutions in octanoic acidDMSO (black) and TCB-DMSO (red). (b) Emission spectra of PDBA solutions in octanoic acid-DMSO (black) and TCB-DMSO (red). (c) Excitation spectra $\left(\lambda_{\text {obs }}=500 \mathrm{~nm}\right.$ ) of PDBA thin films obtained from 1.0 $\times 10^{-4}$ M octanoic acid-DMSO (black) and TCB-DMSO (red). (d) Emission spectra $\left(\lambda_{\text {exc }}=340 \mathrm{~nm}\right.$ ) of PDBA thin films obtained from $1.0 \times 10^{-4}$ M octanoic acid-DMSO (black) and TCB-DMSO (red). 
In order to get further insight into the growth of 2DP films on solid surfaces, dry films obtained after evaporation of the solvent mixtures were also characterized using UV-Vis absorption and emission spectroscopy. Since the HOPG substrates used for STM characterization are strongly absorbing and nontransparent, mica was used as the substrate for these experiments similar to the approach used for the ATR-IR measurements described earlier. The films were allowed to equilibrate overnight and were eventually dried using a heat gun. Figure $5 c$ displays the excitation spectrum obtained on the asdeposited thin films prepared from octanoic acid and TCB. Major changes can be readily noticed upon comparison between the absorption spectra obtained from solutions (Figure 5a) and the excitation spectra $\left(\lambda_{\text {obs }}=500 \mathrm{~nm}\right)$ obtained from thin films (Figure $5 \mathrm{c}$ ) indicating significant difference in the behavior of the system in solution and as thin films.

The excitation spectra of the thin films obtained from octanoic acid-DMSO solution (black, Figure 5c) appears to be a combination of the absorption spectrum of PDBA monomers in octanoic acid-DMSO solution (Figure $5 \mathrm{a}$ ) and a red shifted species $(\sim 396 \mathrm{~nm})$ which could be a signature of the 2DP formed on the mica surface. Thus, thin films deposited on mica from octanoic acid-DMSO solution plausibly contain unreacted PDBA monomers as well as the corresponding 2DP. The same conclusion can be reached by comparing the emission spectra of the PDBA monomers in octanoic acid-DMSO solution (black, Figure 5b) versus those obtained from films (black, Figure 5d). Most importantly, the emission spectrum of thin films obtained from octanoic acid-DMSO solutions is structured and only slightly red shifted $(\sim 10 \mathrm{~nm})$ relative to the emission from solution. This indicates lack of excimer emission.

The excitation spectrum of the thin films obtained from TCB-DMSO solutions (red, Figure 5c) however appears drastically different than that the absorption spectrum of the monomers in TCB-DMSO solution (red, Figure 5a). Specifically, the excitation spectrum $\left(\lambda_{\text {obs }}=500 \mathrm{~nm}\right)$ consists on an unstructured broad band ranging from $350 \mathrm{~nm}$ to $500 \mathrm{~nm}$. Furthermore, the emission spectra of the thin films obtained from TCB-DMSO solution also show a broad unstructured emission with maximum around $455 \mathrm{~nm}$ (Figure 5d). Such unstructured excitation and emission bands suggest aggregation of the pyrene chromophore via $\pi-\pi$ stacking which leads to exciton interaction as well as interaction with charge transfer states. ${ }^{49-52}$ Thus, the emission spectra obtained on the thin films obtained from TCB-DMSO solutions can be considered as a superposition of spectra of isolated monomers and $\pi$-stacked species.

The formation of $\pi$-stacked species yielding excimer-like emission in TCB-DMSO solution was further confirmed by comparing the emission spectra of thin films obtained from both solvents and using $414 \mathrm{~nm}$ excitation (Figure S15 in the supporting information). While a structureless emission band with a maximum at $450 \mathrm{~nm}$ was observed for thin films obtained from TCB-DMSO solution, the emission spectrum of thin films obtained from octanoic acid-DMSO solutions mainly yielded the tail of the vibrational progression. The spectral position of the band observed for the TCB sample is similar to that of excimer emission of pyrene. ${ }^{48}$ 
Finally, a comparison between the emission spectra obtained from fresh and aged solutions revealed a decrease in the intensity of the band corresponding to the $S_{0} \rightarrow S_{1}$ transition for the aged solution upon excitation at $340 \mathrm{~nm}$ and an increase in the intensity of the tail for the aged solution upon excitation at $414 \mathrm{~nm}$ (Figure S16 in the supporting information). The former indicates either a decrease of the absorbance at $340 \mathrm{~nm}\left(\mathrm{~S}_{0} \rightarrow \mathrm{S}_{2}\right.$ transition of PDBA monomers) or a decreased fluorescence quantum yield of the monomer emission (e.g. by energy transfer to $\pi$-stacked species). The latter suggests the presence of emission from a red shifted species in the aged solution. Both factors are compatible with formation of higher order $\pi-\pi$ stacked species upon ageing of the solution. Considering the results of UVVis emission spectroscopy together with the STM results presented in Figure 4, it is not unreasonable to conclude that the initial self-condensation of PDBA leading to nucleation the PDBA 2DP begins in solution when TCB-DMSO combination is used as a solvent.

The indication that the self-condensation of boronic acids is possibly initiated in the solution phase when using TCB as the main solvent should be considered against a previous report where room temperature on-surface synthesis of boronic ester-based 2DPs was reported. ${ }^{53}$ In this report, NMR spectroscopy was used to determine if the polymerization proceeds in the solution phase. The ${ }^{1} \mathrm{H}$ NMR spectra obtained in DMSO- $d_{6}$ revealed that most precursors exist as unreacted monomers in solution even after five days and only a small fraction (17.2\%) was found to transform into the corresponding boronic esters, most probably as dimer or trimers. However, the on-surface 2D polymerization experiments were carried out in a different solvent, namely octanoic acid, similar to the present study. Besides, the present results clearly reveal that the mode of growth of the 2DP could strongly depend on the choice solvent.

\section{CONCLUSIONS}

Despite its popularity, the on-surface synthesis of 2DPs is limited to targeting a given topology, and although rarely discussed in published literature, often plagued by irreproducibility of the synthesis. Due to the lack of reproducibility, there has been limited experimental research on the variables affecting the growth of 2DPs. To alleviate these issues and to formulate rational strategies for 2DP synthesis, it is essential to realize reaction conditions under which the polymerization proceeds at room temperature and under homogenous conditions. This has recently been pointed out for bulk 2D COF as well as onsurface 2DP synthesis. With better reproducibility it would be possible to obtain a clear microscopic picture of the 2DP growth.

In the work presented above, we have taken initial steps to address some of the aforementioned issues. The room temperature synthesis of a boroxine 2DP under homogenous conditions (and not from suspensions) was achieved. The straightforward protocol not only allowed us to characterize the discrete outcomes of the on-surface $2 \mathrm{D}$ polymerization process as a function of monomer concentration, but also the dynamics of the polymerization process could be captured using sequential STM imaging. We show 
that the monomer concentration essentially decides whether a physisorbed film of an extended 2DP, or self-assembled network of covalent trimer or that of monomers is formed at the solution-solid interface. Collectively, the STM and the UV-Vis absorption/emission data indicate that the 2DP in question grows via different modes depending on the choice of the solvent.

While these initial results are highly promising, they represent only the beginning towards understanding the growth of 2DPs. Despite the dynamic polymerization process, the crystalline domain size still appears to be limited to a few hundred nanometers. There is still ample room to improve the domain size of these 2DPs and in this context, recent breakthroughs achieved in the synthesis of bulk 2D COFs are promising. Recently reported strategies such as slow monomer addition and the use of additives for gradual monomer exchange have proved to be extremely fruitful for increasing the size of crystalline 2DCOF particles. These strategies, which reduce the rate of nucleation of the 2DP, also hold potential for on-surface 2DP synthesis and should be adapted for targeting micron size crystalline flakes of 2DPs. The UV-Vis data clearly show the potential spectroscopic methods hold in providing in-depth understanding of 2DP growth in solution and as films. The isolation of monodisperse covalent trimers highlights the strength on-surface synthesis holds for the preparation of reaction products not easily accessible via conventional methods. The insight gained from such systems can readily be transferred to bulk 2D COFs provided a combination of surface analytical tools such as STM and spectroscopic methods is used in a synergistic fashion.

\section{EXPERIMENTAL SECTION}

Materials and general methods. Reagents for synthesis were, if not otherwise specified, purchased from Aldrich, TCI or Acros Organics. Toluene was dried using an Innovative Pure Solve solvent purification system. Column chromatography was carried out using Silica gel $60(40-60 \mu \mathrm{m})$ from Scharlab. Analytical thin layer chromatography (TLC) was done using aluminum sheets $(20 \times 20 \mathrm{~cm})$ precoated with silica gel RP-18W 60 F254 from Merck. UV-active compounds were detected with a UV-lamp from CAMAG at wavelength $\lambda=254$ or $366 \mathrm{~nm}$. NMR spectra in solution were recorded on Bruker Avance 400 spectrometer at room temperature using partially deuterated solvents as internal standards. Coupling constants () are denoted in $\mathrm{Hz}$ and chemical shifts $(\delta)$ in ppm. Matrix Assisted Laser Desorption Ionization (coupled to a Time-Of-Flight analyzer) experiments (MALDI-TOF) were recorded on a Bruker REFLEX spectrometer.

Scanning tunneling microscopy (STM). All STM experiments were performed at room temperature $\left(20-22^{\circ} \mathrm{C}\right)$ using a PicoLE (Keysight) or Molecular Imaging STM system in constant-current mode. STM tips were prepared by mechanical cutting from Pt/Ir wire (80/20, diameter $0.25 \mathrm{~mm}$, Advent Research Materials). Freshly cleaved HOPG (grade ZYB, Momentive Performance Material Quartz Inc., Strongsville, 
OH, USA) and mica substrates (AGG250-1, Agar Scientific Ltd.) were used. STM measurements were performed under ambient conditions and at the HOPG-liquid interface. The STM images presented in the main text are Gaussian filtered. Several samples were investigated, and for each sample, several locations were probed. Imaging parameters for the STM images are indicated in the figure captions and denoted by $V_{\text {bias }}$ for sample bias and $I_{\text {set }}$ for tunneling current. The bias voltage refers to the substrate. The STM images were processed using Scanning Probe Image Processor (SPIP, Image Metrology ApS) software.

Infrared spectroscopy. Fourier Transform-Infrared Spectroscopy (FT-IR) spectra on the bulk solids have been recorded on a Bruker ALPHA ATR-IR spectrometer using solid samples. FT-IR spectroscopy experiments on the supported dry films were performed using a VERTEX 70 FTIR spectrometer from Bruker operated with attenuated total reflection sampling technique.

Computational methods. The lattice parameters were calculated using plane-wave periodic Density Functional Theory. Initial atomic structures were prepared based on the experimental lattice parameters and STM images. The effect of the graphite substrate and the solvent on the lattice parameter were assumed to be negligible, and hence the prepared structures (PDBA trimer assembly, PDBA-2DP, and PDBA monomer assembly) are periodic and free-standing. Optimization of the lattice parameters and atomic positions was carried out using the vdW-DF long-range functional as implemented in Quantum ESPRESSO, in order to adequately account for intermolecular interactions. The core electrons were modelled using PAW potentials, and the density and wavefunction cut-offs were set up respectively to 350. and 48. Ry. The gamma point energies were converged to $10^{-6} \mathrm{Ry}$. The hydrogen bonding complexation energy was calculated using a gas-phase PDBA dimer and monomer. Spurious interactions between dimers were avoided by using the Martyna-Tuckerman correction. The dimerization energy (complexation and reorganization energy) for a dimer of PDBA is $-7.08 \mathrm{kcal} / \mathrm{mol}$ as calculated with Quantum ESPRESSO. A PBE0-D3/cc-pdVDZ calculation including BSSE with Gaussian 16 renders a slightly larger dimerization energy, $-10.8 \mathrm{kcal} / \mathrm{mol}$. The PDBA molecular orbitals and electrostatic potential were obtained a PBE0-D3/cc-pVDZ optimization carried out with Gaussian 16. The calculated STM images of PDBA on graphene were calculated with periodic DFT. Atomic structures of a PDBA molecule adsorbed on a $17.04 \times 17.22 \AA$ periodic graphene layer were optimized at the gamma-point using the vdW-DF2 functional as implemented in SIESTA, a double-zeta basis set with polarization, normconserving pseudopotentials, a PAO energy shift of 0.01 Ry and real space integration mesh cut-off of 220 Ry. A slab-to-slab space of 25 AA was used to avoid spurious interactions. STM images were simulated using the Tersoff-Hammann approximation at constant-current and various bias voltages using a $5 \times 5 \times$ 1 k-point grid. 
UV-visible absorption and emission measurements. The UV-visible steady-state absorption and emission spectra were recorded using a Cary 100 Bio UV-visible or Lamda-950 spectrometer and a FLS920 Spectrofluorometer (Edinburgh Instrument Ltd, Livingston, UK), respectively. The Edinburgh FLS920 spectrometer was corrected for the wavelength dependence of the throughput of the emission monochromator and the sensitivity of the detector.

Synthetic details. Pyrene-2,7-diboronic acid (PDBA) and pyrene-2-boronic acid (PMBA) were synthesized according to reported methods. ${ }^{54}$ The bulk pyrene-2,7-diboronic acid 2D COF was synthesized using a reported method. ${ }^{33}$

Synthesis of model trimer T. The model trimer was synthesized from pyrene-2-boronic acid (PMBA). PMBA was refluxed in toluene using a Dean-Stark setup. After 7 hours the reaction was cooled down to room temperature and the solvent evaporated, giving rise to a white solid. ${ }^{1} \mathrm{H}$ NMR $(400 \mathrm{MHz}, 1,1,2,2$ tetrachloroethane- $\left.\mathrm{d}_{2}\right)$ ): $9.31(\mathrm{~s}, 6 \mathrm{H}), 8.45(\mathrm{~d}, J=8.9 \mathrm{~Hz}, 6 \mathrm{H}), 8.31(\mathrm{~d}, J=8.3 \mathrm{~Hz}, 6 \mathrm{H}), 8.25(\mathrm{~d}, J=8.3 \mathrm{~Hz}, 6 \mathrm{H})$, $8.18-8.08(\mathrm{~m}, 3 \mathrm{H})$. Due to its poor solubility, a ${ }^{13} \mathrm{C}$ NMR spectrum with acceptable signal to noise ratio could not be obtained. EM (MALDI-TOF) (m/z): calculated for $\mathrm{C}_{48} \mathrm{H}_{27} \mathrm{~B}_{3} \mathrm{O}_{3}: 684.224$; found: 684.145 [M] . FT-IR (cm-1): 1559.1, 1461.0, 1346.1, 1315.8, 1217.2, 1176.9, 887.0, 838.3, 811.6, 721.0, 629.5 (total disappearance of the hydroxyl stretching frequencies at $3285 \mathrm{~cm}^{-1}$ of pyrene-2-boronic acid).

On-surface synthesis of PDBA 2DP. The protocol followed to prepare the PDBA solutions and their deposits on HOPG was adapted from a previously reported procedure for the on-surface synthesis of imine-based 2DPs on bare HOPG. 40, 55 Owing to the limited solubility of the chemicals in octanoic acid (OA) or 1,2,4trichlorobenzene (TCB), all building blocks were first dissolved in dimethyl sulfoxide (DMSO, >99.0\% Sigma-Aldrich), and then, the solution was diluted in OA (>98.0\% TCI) or TCB ( $>99.0 \%$ Sigma-Aldrich) to a designated concentration, indicated in each case. Note that DMSO volume content in the different prepared solutions range between $0.3-10 \%\left(0.3 \%\right.$ for $1.0 \times 10^{-5} \mathrm{M}$; $0.45 \%$ for $1.5 \times 10^{-5} \mathrm{M}$; $1.3 \%$ for 5.0 $\times 10^{-5} \mathrm{M} ; 2.6 \%$ for $1.0 \times 10^{-4} \mathrm{M}$ and $10 \%$ for $3.8 \times 10^{-4} \mathrm{M}$ ). $5 \mu \mathrm{L}$ of the final solution was drop-casted on the freshly cleaved HOPG surface at room temperature. The resulting sample was characterized by STM.

\section{ASSOCIATED CONTENT}

The authors declare no competing financial interests.

\section{Supporting Information}

The Supporting Information is available free of charge on the ACS Publications website at DOI:XXXXXXXX.

Experimental details and additional data: STM and AFM images; NMR, UV-vis and IR spectra (PDF)

\section{AUTHOR INFORMATION}




\section{Corresponding Authors}

*nerea.bilbao@kuleuven.be

*kunal.mali@kuleuven.be

*steven.defeyter@kuleuven.be

\section{ORCID}

Nerea Bilbao: 0000-0001-7368-3322

Kunal S. Mali: 0000-0002-9938-6446

Steven De Feyter: 0000-0002-0909-9292

\section{Author Contributions}

$\S$ C.M. and G.Z. contributed equally

\section{ACKNOWLEDGMENTS}

This work was financially supported by KU Leuven Internal Funds and the Fund of Scientific ResearchFlanders (FWO). N. B. acknowledges the European Union's Horizon 2020 programme for Marie Skłodowska-Curie Individual Fellowships (Project ID 741384, CustomCOF). C.M. thanks the FWO for the fellowships received (12J1719N and 12J1716N). A.M.A is are grateful to the Basque Science Foundation for Science (Ikerbasque), POLYMAT, the University of the Basque Country (Grupo de Investigación GIU17/054 and SGIker), Gobierno de España (Ministerio de Economía y Competitividad CTQ201677970-R), Gobierno Vasco (BERC programme) and Diputación Foral de Guipúzcoa (OF215/2016(ES)). This project has received funding from the European Research Council (ERC) under the European Union's Horizon 2020 research and innovation programme (grant agreement no. 722951). This project has received funding from the European Union's Horizon 2020 research and innovation programme under grant agreement No 664878.

\section{References:}

1. Feng, X.; Schlüter, A. D., Towards Macroscopic Crystalline 2D Polymers. Angew. Chem. Int. Ed. 2018, 57, 13748-13763.

2. Servalli, M.; Öttinger, H. C.; Schlüter, A. D., Synthesizing Molecular Fishing Nets. Phys. Today 2018, $71,40-47$.

3. Liu, X.-H.; Guan, C.-Z.; Wang, D.; Wan, L.-J., Graphene-Like Single-Layered Covalent Organic Frameworks: Synthesis Strategies and Application Prospects. Adv. Mater. 2014, 26, 6912-6920.

4. Segura, J. L.; Mancheno, M. J.; Zamora, F., Covalent Organic Frameworks Based on Schiff-Base Chemistry: Synthesis, Properties and Potential Applications. Chem. Soc. Rev. 2016, 45, 5635-5671. 
5. Dienstmaier, J. F.; Gigler, A. M.; Goetz, A. J.; Knochel, P.; Bein, T.; Lyapin, A.; Reichlmaier, S.; Heckl, W. M.; Lackinger, M., Synthesis of Well-Ordered COF Monolayers: Surface Growth of Nanocrystalline Precursors versus Direct On-Surface Polycondensation. ACS Nano 2011, 5, 9737-9745.

6. $\quad$ Cai, Z.-F.; Zhan, G.; Daukiya, L.; Eyley, S.; Thielemans, W.; Severin, K.; De Feyter, S., Electric-FieldMediated Reversible Transformation between Supramolecular Networks and Covalent Organic Frameworks. J. Am. Chem. Soc. 2019, 141, 11404-11408.

7. Colson, J. W.; Woll, A. R.; Mukherjee, A.; Levendorf, M. P.; Spitler, E. L.; Shields, V. B.; Spencer, M. G.; Park, J.; Dichtel, W. R., Oriented 2D Covalent Organic Framework Thin Films on Single-Layer Graphene. Science 2011, 332, 228-231.

8. Martínez-Abadía, M.; Stoppiello, C. T.; Strutynski, K.; Lerma-Berlanga, B.; Martí-Gastaldo, C.; Saeki, A.; Melle-Franco, M.; Khlobystov, A. N.; Mateo-Alonso, A., A Wavy Two-Dimensional Covalent Organic Framework from Core-Twisted Polycyclic Aromatic Hydrocarbons. J. Am. Chem. Soc. 2019, 141, 1440314410.

9. Liu, X.-H.; Guan, C.-Z.; Ding, S.-Y.; Wang, W.; Yan, H.-J.; Wang, D.; Wan, L.-J., On-Surface Synthesis of Single-Layered Two-Dimensional Covalent Organic Frameworks via Solid-Vapor Interface Reactions. J. Am. Chem. Soc. 2013, 135, 10470-10474.

10. Lackinger, M., Surface-Assisted Ullmann Coupling. Chem. Commun. 2017, 53, 7872-7885.

11. Murray, D. J.; Patterson, D. D.; Payamyar, P.; Bhola, R.; Song, W.; Lackinger, M.; Schlüter, A. D.; King, B. T., Large Area Synthesis of a Nanoporous Two-Dimensional Polymer at the Air/Water Interface. J. Am. Chem. Soc. 2015, 137, 3450-3453.

12. Kandambeth, S.; Dey, K.; Banerjee, R., Covalent Organic Frameworks: Chemistry beyond the Structure. J. Am. Chem. Soc. 2019, 141, 1807-1822.

13. Berlanga, I.; Ruiz-González, M. L.; González-Calbet, J. M.; Fierro, J. L. G.; Mas-Ballesté, R.; Zamora, F., Delamination of Layered Covalent Organic Frameworks. Small 2011, 7, 1207-1211.

14. Marco, A. B.; Cortizo-Lacalle, D.; Perez-Miqueo, I.; Valenti, G.; Boni, A.; Plas, J.; Strutyński, K.; De Feyter, S.; Paolucci, F.; Montes, M.; Khlobystov, A. N.; Melle-Franco, M.; Mateo-Alonso, A., Twisted Aromatic Frameworks: Readily Exfoliable and Solution-Processable Two-Dimensional Conjugated Microporous Polymers. Angew. Chem. Int. Ed. 2017, 129, 7050-7055.

15. Lackinger, M., On-Surface Polymerization - A Versatile Synthetic Route to Two-Dimensional Polymers. Polym. Int. 2015, 64, 1073-1078.

16. Steiner, C.; Gebhardt, J.; Ammon, M.; Yang, Z.; Heidenreich, A.; Hammer, N.; Görling, A.; Kivala, M.; Maier, S., Hierarchical On-Surface Synthesis and Electronic Structure of Carbonyl-Functionalized Oneand Two-Dimensional Covalent Nanoarchitectures. Nat. Commun. 2017, 8, 14765.

17. Plas, J.; Ivasenko, O.; Martsinovich, N.; Lackinger, M.; De Feyter, S., Nanopatterning of a Covalent Organic Framework Host-Guest System. Chem. Commun. 2016, 52, 68-71. 
18. Cui, D.; MacLeod, J. M.; Ebrahimi, M.; Perepichka, D. F.; Rosei, F., Solution and Air Stable Host/Guest Architectures from a Single Layer Covalent Organic Framework. Chem. Commun. 2015, 51, 16510-16513.

19. Dienstmaier, J. F.; Medina, D. D.; Dogru, M.; Knochel, P.; Bein, T.; Heckl, W. M.; Lackinger, M., Isoreticular Two-Dimensional Covalent Organic Frameworks Synthesized by On-Surface Condensation of Diboronic Acids. ACS Nano 2012, 6, 7234-7242.

20. Yue, J.-Y.; Mo, Y.-P.; Li, S.-Y.; Dong, W.-L.; Chen, T.; Wang, D., Simultaneous Construction of Two Linkages for the On-Surface Synthesis of Imine-Boroxine Hybrid Covalent Organic Frameworks. Chem. Sci. 2017, 8, 2169-2174.

21. Müller, V.; Hinaut, A.; Moradi, M.; Baljozovic, M.; Jung, T. A.; Shahgaldian, P.; Möhwald, H.; Hofer, G.; Kröger, M.; King, B. T.; Meyer, E.; Glatzel, T.; Schlüter, A. D., A Two-Dimensional Polymer Synthesized at the Air/Water Interface. Angew. Chem. Int. Ed. 2018, 57, 10584-10588.

22. Liu, K.; Qi, H.; Dong, R.; Shivhare, R.; Addicoat, M.; Zhang, T.; Sahabudeen, H.; Heine, T.; Mannsfeld, S.; Kaiser, U.; Zheng, Z.; Feng, X., On-Water Surface Synthesis of Crystalline, Few-Layer Two-Dimensional Polymers Assisted by Surfactant Monolayers. Nat. Chem. 2019, 11, 994-1000.

23. Shao, F.; Dai, W.; Zhang, Y.; Zhang, W.; Schlüter, A. D.; Zenobi, R., Chemical Mapping of Nanodefects within 2D Covalent Monolayers by Tip-Enhanced Raman Spectroscopy. ACS Nano 2018, 12, 5021-5029. 24. Li, H.; Chavez, A. D.; Li, H.; Li, H.; Dichtel, W. R.; Bredas, J.-L., Nucleation and Growth of Covalent Organic Frameworks from Solution: The Example of COF-5. J. Am. Chem. Soc. 2017, 139, 16310-16318.

25. Smith, B. J.; Dichtel, W. R., Mechanistic Studies of Two-Dimensional Covalent Organic Frameworks Rapidly Polymerized from Initially Homogenous Conditions. J. Am. Chem. Soc. 2014, 136, 8783-8789.

26. Li, Y.; Chen, Q.; Xu, T.; Xie, Z.; Liu, J.; Yu, X.; Ma, S.; Qin, T.; Chen, L., De Novo Design and Facile Synthesis of 2D Covalent Organic Frameworks: A Two-in-One Strategy. J. Am. Chem. Soc. 2019, 141, $13822-13828$.

27. Yan, X.; Liu, H.; Li, Y.; Chen, W.; Zhang, T.; Zhao, Z.; Xing, G.; Chen, L., Ultrastable Covalent Organic Frameworks via Self-Polycondensation of an A2B2 Monomer for Heterogeneous Photocatalysis. Macromolecules 2019, 52, 7977-7983.

28. Koo, B. T.; Heden, R. F.; Clancy, P., Nucleation and Growth of 2D Covalent Organic Frameworks: Polymerization and Crystallization of COF Monomers. Phys. Chem. Chem. Phys. 2017, 19, 9745-9754.

29. Nguyen, V.; Grünwald, M., Microscopic Origins of Poor Crystallinity in the Synthesis of Covalent Organic Framework COF-5. J. Am. Chem. Soc. 2018, 140, 3306-3311.

30. Auras, F.; Ascherl, L.; Hakimioun, A. H.; Margraf, J. T.; Hanusch, F. C.; Reuter, S.; Bessinger, D.; Döblinger, M.; Hettstedt, C.; Karaghiosoff, K.; Herbert, S.; Knochel, P.; Clark, T.; Bein, T., Synchronized Offset Stacking: A Concept for Growing Large-Domain and Highly Crystalline 2D Covalent Organic Frameworks. J. Am. Chem. Soc. 2016, 138, 16703-16710. 
31. Chen, X.; Huang, N.; Gao, J.; Xu, H.; Xu, F.; Jiang, D., Towards Covalent Organic Frameworks with Predesignable and Aligned Open Docking Sites. Chem. Commun. 2014, 50, 6161-6163.

32. Rabbani, M. G.; Sekizkardes, A. K.; Kahveci, Z.; Reich, T. E.; Ding, R.; El-Kaderi, H. M., A 2D Mesoporous Imine-Linked Covalent Organic Framework for High Pressure Gas Storage Applications. Chem. Eur. J 2013, 19, 3324-3328.

33. Wan, S.; Guo, J.; Kim, J.; Ihee, H.; Jiang, D., A Photoconductive Covalent Organic Framework: SelfCondensed Arene Cubes Composed of Eclipsed 2D Polypyrene Sheets for Photocurrent Generation. Angew. Chem. Int. Ed. 2009, 48, 5439-5442.

34. Lin, G.; Ding, H.; Yuan, D.; Wang, B.; Wang, C., A Pyrene-Based, Fluorescent Three-Dimensional Covalent Organic Framework. J. Am. Chem. Soc. 2016, 138, 3302-3305.

35. Stegbauer, L.; Zech, S.; Savasci, G.; Banerjee, T.; Podjaski, F.; Schwinghammer, K.; Ochsenfeld, C.; Lotsch, B. V., Tailor-Made Photoconductive Pyrene-Based Covalent Organic Frameworks for Visible-Light Driven Hydrogen Generation. Adv. Energy Mater. 2018, 8, 1703278.

36. Schlögl, S.; Sirtl, T.; Eichhorn, J.; Heckl, W. M.; Lackinger, M., Synthesis of Two-Dimensional Phenylene-Boroxine Networks through in Vacuo Condensation and On-Surface Radical Addition. Chem. Commun. 2011, 47, 12355-12357.

37. Zwaneveld, N. A. A.; Pawlak, R.; Abel, M.; Catalin, D.; Gigmes, D.; Bertin, D.; Porte, L., Organized Formation of 2D Extended Covalent Organic Frameworks at Surfaces. J. Am. Chem. Soc. 2008, 130, 66786679.

38. Ourdjini, O.; Pawlak, R.; Abel, M.; Clair, S.; Chen, L.; Bergeon, N.; Sassi, M.; Oison, V.; Debierre, J.-M.; Coratger, R.; Porte, L., Substrate-Mediated Ordering and Defect Analysis of a Surface Covalent Organic Framework. Phys. Rev. B 2011, 84, 125421.

39. Côté, A. P.; El-Kaderi, H. M.; Furukawa, H.; Hunt, J. R.; Yaghi, O. M., Reticular Synthesis of Microporous and Mesoporous 2D Covalent Organic Frameworks. J. Am. Chem. Soc. 2007, 129, 1291412915.

40. Yu, Y.; Lin, J.; Wang, Y.; Zeng, Q.; Lei, S., Room Temperature On-Surface Synthesis of TwoDimensional Imine Polymers at the Solid/Liquid Interface: Concentration Takes Control. Chem. Commun. 2016, 52, 6609-6612.

41. Fournier, J.-H.; Maris, T.; Wuest, J. D.; Guo, W.; Galoppini, E., Molecular Tectonics. Use of the Hydrogen Bonding of Boronic Acids To Direct Supramolecular Construction. J. Am. Chem. Soc. 2003, 125, 1002-1006.

42. Fan, Q.; Wang, C.; Han, Y.; Zhu, J.; Hieringer, W.; Kuttner, J.; Hilt, G.; Gottfried, J. M., Surface-Assisted Organic Synthesis of Hyperbenzene Nanotroughs. Angew. Chem. Int. Ed. 2013, 52, 4668-4672.

43. Kaiser, K.; Scriven, L. M.; Schulz, F.; Gawel, P.; Gross, L.; Anderson, H. L., An Sp-Hybridized Molecular Carbon Allotrope, Cyclo[18]Carbon. Science 2019, 365, 1299-1301. 
44. Côté, A. P.; Benin, A. I.; Ockwig, N. W.; O'Keeffe, M.; Matzger, A. J.; Yaghi, O. M., Porous, Crystalline, Covalent Organic Frameworks. Science 2005, 310, 1166-1170.

45. Kuamit, T.; Ratanasak, M.; Rungnim, C.; Parasuk, V., Effects of Shape, Size, and Pyrene Doping on Electronic Properties of Graphene Nanoflakes. J. Mol. Model. 2017, 23, 355.

46. Tzeli, D.; Theodorakopoulos, G.; Petsalakis, I. D.; Ajami, D.; Rebek, J., Theoretical Study of Hydrogen Bonding in Homodimers and Heterodimers of Amide, Boronic Acid, and Carboxylic Acid, Free and in Encapsulation Complexes. J. Am. Chem. Soc. 2011, 133, 16977-16985.

47. Duhamel, J., Internal Dynamics of Dendritic Molecules Probed by Pyrene Excimer Formation. Polymers 2012, 4, 211-239.

48. Crawford, A. G.; Dwyer, A. D.; Liu, Z.; Steffen, A.; Beeby, A.; Pålsson, L.-O.; Tozer, D. J.; Marder, T. B., Experimental and Theoretical Studies of the Photophysical Properties of 2- and 2,7-Functionalized Pyrene Derivatives. J. Am. Chem. Soc. 2011, 133, 13349-13362.

49. Klessinger, M.; Michl, J., Excited States and Photochemistry of Organic Molecules. Wiley-VCH: New York, 1995.

50. Beens, H.; Weller, A., In Organic Molecular Photophysics, Birks, J. B., Ed. Wiley: New York, 1975; pp 159-215.

51. Birks, J. B.; Kazzaz, A. A., Excimer Fluorescence XII. The Pyrene Crystal Excimer Interaction Potential. Proc. R. Soc. Lond. A. 1968, 304, 291-301.

52. Barnes, R. L.; Birks, J. B., 'Excimer' Fluorescence X. Spectral Studies of 9-Methyl and 9,10-Dimethyl Anthracene. Proc. Roy. Soc. A 1966, 291, 570-582.

53. Liu, C.; Yu, Y.; Zhang, W.; Zeng, Q.; Lei, S., Room-Temperature Synthesis of Covalent Organic Frameworks with a Boronic Ester Linkage at the Liquid/Solid Interface. Chem. Eur. J 2016, 22, 1841218418.

54. Crawford, A. G.; Liu, Z.; Mkhalid, I. A. I.; Thibault, M.-H.; Schwarz, N.; Alcaraz, G.; Steffen, A.; Collings, J. C.; Batsanov, A. S.; Howard, J. A. K.; Marder, T. B., Synthesis of 2- and 2,7-Functionalized Pyrene Derivatives: An Application of Selective C-H Borylation. Chem. Eur. J 2012, 18, 5022-5035.

55. Bilbao, N.; Yu, Y.; Verstraete, L.; Lin, J.; Lei, S.; De Feyter, S., The Impact of Grafted Surface Defects on the On-Surface Schiff-Base Chemistry at the Solid-Liquid Interface. Chem. Commun. 2018, 54, 99059908. 
TOC Graphic:

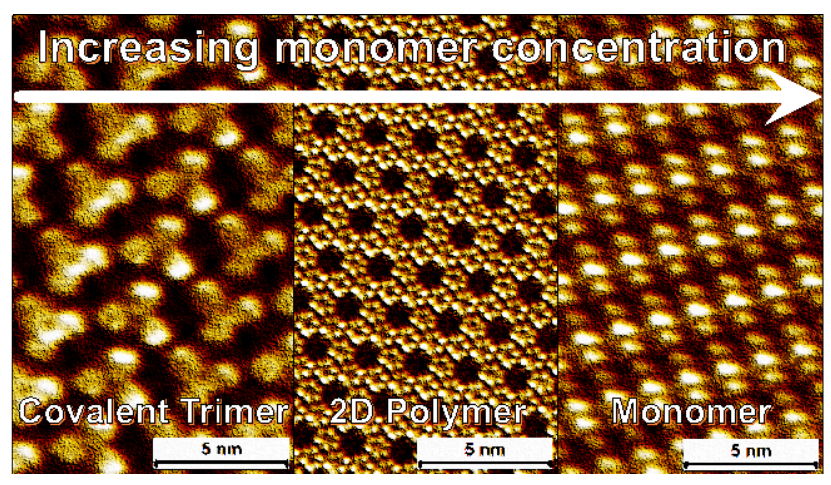

